\title{
A Unique "Nonnegative" Solution to an Underdetermined System: from Vectors to Matrices
}

\author{
Meng Wang Weiyu Xu Ao Tang
}

\begin{abstract}
This paper investigates the uniqueness of a nonnegative vector solution and the uniqueness of a positive semidefinite matrix solution to underdetermined linear systems. A vector solution is the unique solution to an underdetermined linear system only if the measurement matrix has a row-span intersecting the positive orthant. Focusing on two types of binary measurement matrices, Bernoulli 0-1 matrices and adjacency matrices of general expander graphs, we show that, in both cases, the support size of a unique nonnegative solution can grow linearly, namely $O(n)$, with the problem dimension $n$. We also provide closed-form characterizations of the ratio of this support size to the signal dimension. For the matrix case, we show that under a necessary and sufficient condition for the linear compressed observations operator, there will be a unique positive semidefinite matrix solution to the compressed linear observations. We further show that a randomly generated Gaussian linear compressed observations operator will satisfy this condition with overwhelmingly high probability.
\end{abstract}

\section{INTRODUCTION}

This paper is devoted to recover a "nonnegative" decision variable from an underdetermined system of linear equations. When the decision variable is a vector, "nonnegativity" means each entry is nonnegative. When the decision variable is a matrix, "nonnegativity" indicates that the matrix is positive semidefinite. The problem is ill-conditioned in general, however, we can correctly recover the vector or the matrix if the vector is sparse, or the matrix is low rank.

Finding the sparest vector among vectors satisfying a set of linear equations is NP-hard [7]. One frequently used heuristic is $\ell_{1}$-minimization, which returns the vector with the least $\ell_{1}$ norm. Recently, there has been an explosion of research on this topic, see e.g., [2], [7]-[9], [14]. [7] gives a sufficient condition known as Restricted Isometry Property (RIP) on the measurement matrix that guarantees the recovery of the sparest vector via $\ell_{1}$ minimization. In many interesting cases, the vector to recover is nonnegative [5][12][31]. [12] gives a necessary and sufficient condition known as the outwardly neighborliness property of the measurement matrix for $\ell_{1}$ minimization to successfully recover a sparse nonnegative vector. Moreover, recent studies [5], [13], [20] suggested that a sparse solution could be the unique nonnegative solution. This can potentially lead to better alternatives to $\ell_{1}$ minimization as in this case any optimization problem (with any objective function, for example, $\ell_{2}$ norm) over this constraint set can recover the original unknown. In addition, the sparsest solution can be viewed as a biased solution to an underdetermined

The authors are with School of Electrical and Computer Engineering, Cornell University, Ithaca, NY, 14853. \{mw467, wx42, at422\}@cornell.edu. system, which is undesired in the unbiased networks diagnosis [31]. However, if the uniqueness property holds, the sparse solution is indeed the only nonnegative solution, and thus, unbiased. Therefore, the uniqueness property could be useful in providing unbiased networks diagnosis.

Motivated by networking inference problems such as network tomography, we are particularly interested in systems where the measurement matrix is a 0-1 matrix. There have not been many existing results on this type of systems except a few very recent papers [3], [4], [20], [29]. We focus on two types of binary matrices, Bernoulli 0-1 matrices and adjacency matrices of expanders, and provide conditions under which a sparse vector is the unique nonnegative solution to the underdetermined system. For random Bernoulli measurement matrices, we prove that, as long as the number of equations divided by the number of variables remains constant as the problem dimension grows, with overwhelming probability over the choices of matrices, a sparse nonnegative vector is a unique nonnegative solution provided that its support size is at most proportional to its dimension for some positive ratio. For general expander matrices, we further provide a closedform constant ratio of support size to dimension under which a nonnegative vector is the unique solution.

The phenomenon that an underdetermined system admits a unique "nonnegative" solution is not restricted for the vector case. Finding the minimum rank matrix among all matrices satisfying given linear equations is a rank minimization problem. Among the rank minimization problems, one particularly important class is the rank minimization problem for positive semidefinite matrices under compressed observations. For example, minimizing the rank of a covariance matrix, which is a positive semidefinite matrix, arises in statistics, econometrics, signal processing and many other fields where second-order statistics for random processes are used [16]. A positive semidefinite matrix is special in that its eigenvalues (also its singular values) are nonnegative. In fact, the nuclear norm minimization heuristic for general matrices was preceded by the trace norm heuristic for positive symmetric matrices in rank minimization problems. While the general analytic frameworks and computational techniques, for example, [25], [26], are applicable to the rank minimization problems for positive semidefinite matrices, the special properties of positive semidefinite matrices may open the way to new structures and new analysis, which more efficient computational techniques may exploit to provide faster matrix recovery.

Parallel to the influence of the nonnegative constraint on a vector variable, the positive semidefinite constraint on a matrix variable may dramatically reduce the size of the feasible set 
in rank minimization problems. In particular, we show that under a necessary and sufficient condition for the linear compressed observations operator, there will be a unique positive semidefinite matrix solution to compressed linear observations. We further show that a randomly generated Gaussian linear compressed observations operator will satisfy this necessary and sufficient condition with overwhelmingly high probability. This result is akin to the one in the vector case for the unique nonnegative solution, but the transition from a nonnegative vector to a positive semidefinite matrix requires very different analytical approaches.

This paper is organized as follows. Section II discusses the phenomena that a sparse vector can be the unique nonnegative vector satisfying an underdetermined linear system. Focusing on 0-1 matrices, we prove that a sparse vector is a unique nonnegative solution as long as its support size is at most proportional to the dimension for some positive ratio. We further give a closed-form ratio of the support size and the dimension if the matrix is an adjacent matrix of an expander graph. Section III shows a low-rank matrix can be the unique positive semidefinite matrix satisfying compressed linear measurements. We provide a necessary and sufficient condition for this phenomenon to happen and prove the existence of compressed measurements satisfying the proposed condition. Numerical examples are discussed in Section IV and Section $\mathrm{V}$ concludes the paper.

\section{UniQUE NONNEGATIVE VECTOR TO AN UNDERDETERMINED SYSTEM}

How to recover a vector $\mathbf{x} \in \mathbb{R}^{n}$ from the measurement $\mathbf{y}=$ $A \mathrm{x} \in \mathbb{R}^{m}$, where $A^{m \times n}(m<n)$ is the measurement matrix? In many applications, $\mathbf{x}$ is nonnegative, which is our main focus here. In general, the task seems impossible as we have fewer measurements than variables. However, if $\mathbf{x}$ is sparse, it can be recovered by solving the following problem,

$$
\min \|\mathbf{x}\|_{0} \quad \text { s.t. } A \mathbf{x}=\mathbf{y}, \mathbf{x} \geq \mathbf{0},
$$

where the $\ell_{0}$ norm $\|\cdot\|_{0}$ measures the number of nonzero entries of a given vector. Since (II.1) in general is NP-hard, people solve an alternative convex problem by replacing $\ell_{0}$ norm with $\ell_{1}$ norm where $\|\mathbf{x}\|_{1}=\sum_{i}\left|x_{i}\right|$.The $\ell_{1}$ minimization problem can be formulated as follows:

$$
\min \mathbf{1}^{T} \mathbf{x} \quad \text { s.t. } A \mathbf{x}=\mathbf{y}, \mathbf{x} \geq \mathbf{0} .
$$

In fact, for a certain class of matrices, if $\mathbf{x}$ is sufficiently sparse, not only can we recover $\mathbf{x}$ from (II.2), but also $\mathbf{x}$ is the only solution to $\{\mathbf{x} \mid A \mathbf{x}=\mathbf{y}, \mathbf{x} \geq \mathbf{0}\}$. In other words, $\{\mathbf{x} \mid A \mathbf{x}=\mathbf{y}, \mathbf{x} \geq \mathbf{0}\}$ is a singleton. Then $\mathbf{x}$ can possibly be recovered by other techniques to be developed besides $\ell_{1}$ minimization, since in this case the set $\{\mathbf{x} \mid A \mathbf{x}=\mathbf{y}, \mathbf{x} \geq$ 0) contains only one solution, which can be recovered by optimizing any objective function over this constraint set.

[5] analyzed the singleton property of matrices with a rowspan intersecting the positive orthant. Here we first show only these matrices can possibly have the singleton property.

Definition 1 ([5]). A has a row-span intersecting the positive orthant, denoted by $A \in \mathbf{M}^{+}$, if $\exists \boldsymbol{\beta}>\mathbf{0}\left(\boldsymbol{\beta} \in \mathbb{R}^{n}\right)$ in the row space of $A$, i.e. $\exists \mathbf{h} \in \mathbb{R}^{m}$ such that $\mathbf{h}^{T} A=\boldsymbol{\beta}^{T}>\mathbf{0}$.
There is a simple observation regarding matrices in $\mathbf{M}^{+}$.

Lemma 1. Let $\mathbf{a}_{i} \in \mathbb{R}^{m}(i=1,2, \ldots, n)$ be the $i^{\text {th }}$ column of matrix $A$, then $A \in \mathbf{M}^{+}$if and only if $\mathbf{0} \notin P$, where

$P \triangleq \boldsymbol{C o n v}\left(\mathbf{a}_{1}, \mathbf{a}_{2}, \ldots, \mathbf{a}_{n}\right)=\left\{\sum_{i} \lambda_{i} \mathbf{a}_{i} \mid \mathbf{1}^{T} \boldsymbol{\lambda}=1, \boldsymbol{\lambda} \geq \mathbf{0}, \boldsymbol{\lambda} \in \mathbb{R}^{n}\right\}$

Proof: If $A \in \mathbf{M}^{+}$, then $\exists \mathbf{h} \in \mathbb{R}^{m}$ such that $\mathbf{h}^{T} A=$ $\boldsymbol{\beta}^{T}>\mathbf{0}$. Suppose we also have $\mathbf{0} \in P$, then $\exists \boldsymbol{\lambda} \geq \mathbf{0}\left(\boldsymbol{\lambda} \in \mathbb{R}^{n}\right)$ such that $A \boldsymbol{\lambda}=\mathbf{0}$ and $\mathbf{1}^{T} \boldsymbol{\lambda}=1$. Then $\left(\mathbf{h}^{T} A\right) \boldsymbol{\lambda}=\boldsymbol{\beta}^{T} \boldsymbol{\lambda}>0$ as $\boldsymbol{\beta}>\mathbf{0}, \boldsymbol{\lambda} \geq \mathbf{0}$ and $\boldsymbol{\lambda} \neq \mathbf{0}$. But $\left(\mathbf{h}^{T} A\right) \boldsymbol{\lambda}=\mathbf{h}^{T}(A \boldsymbol{\lambda})=0$ as $A \boldsymbol{\lambda}=\mathbf{0}$. Contradiction! Therefore $\mathbf{0} \notin P$.

Conversely, if $\mathbf{0} \notin P$, there exists a separating hyperplane $\left\{\mathbf{x} \mid \mathbf{h}^{T} \mathbf{x}+b=0, \mathbf{h} \neq \mathbf{0}\right\}$ that strictly separates $\mathbf{0}$ and $P$. We assume without loss of generality that $\mathbf{h}^{T} \mathbf{0}+b<0$ and $\mathbf{h}^{T} \mathbf{x}+b>0$ for any point $\mathbf{x}$ in $P$. Then $\mathbf{h}^{T} \mathbf{a}_{i}>-b>0, \forall i$. Thus we conclude $\mathbf{h}^{T} A>\mathbf{0}$.

The next theorem states a necessary condition on matrix $A$ for $\left\{\mathbf{x} \mid A \mathbf{x}=A \mathbf{x}_{0}, \mathbf{x} \geq \mathbf{0}\right\}$ to be a singleton.

Theorem 1. If $\left\{\mathbf{x} \mid A \mathbf{x}=A \mathbf{x}_{0}, \mathbf{x} \geq \mathbf{0}\right\}$ is a singleton for some $\mathbf{x}_{0} \geq \mathbf{0}$, then $A \in \mathbf{M}^{+}$.

Proof: Suppose $A \notin \mathbf{M}^{+}$, from Lemma 1 we know $\mathbf{0} \in$ $\operatorname{Conv}\left(a_{1}, a_{2}, \ldots, a_{n}\right)$. Then $\exists \mathbf{w} \geq \mathbf{0}\left(\mathbf{w} \in \mathbb{R}^{n}\right)$ such that $A \mathbf{w}=$ $\mathbf{0}$ and $\mathbf{1}^{T} \mathbf{w}=1$. Clearly $\mathbf{w} \in \mathbf{N u l l}(A)$ and $\mathbf{w} \neq \mathbf{0}$. Then for any $\gamma>0$ we have $A\left(\mathbf{x}_{0}+\gamma \mathbf{w}\right)=A \mathbf{x}_{0}+\gamma A \mathbf{w}=A \mathbf{x}_{0}$, and $\mathbf{x}_{0}+\gamma \mathbf{w} \geq \mathbf{0}$ provided $\mathbf{x}_{0} \geq \mathbf{0}$. Hence $\mathbf{x}_{0}+\gamma \mathbf{w} \in\{\mathbf{x} \mid A \mathbf{x}=$ $\left.A \mathbf{x}_{0}, \mathbf{x} \geq \mathbf{0}\right\}$.

Theorem 1 shows that $A \in \mathbf{M}^{+}$is a necessary condition for an underdetermined system to admit a unique nonnegative vector. If $A^{m \times n}$ is a random matrix such that every entry is independently sampled from Gaussian distribution with zero mean, then the probability that $\mathbf{0}$ lies in the convex hull of the column vectors of $A$, or equivalently $\left\{\mathbf{x} \mid A \mathbf{x}=A \mathbf{x}_{0}, \mathbf{x} \geq \mathbf{0}\right\}$ is not a singleton for any $\mathbf{x}_{0} \geq \mathbf{0}$, is $1-2^{-n+1} \sum_{k=0}^{m-1}\left(\begin{array}{c}n-1 \\ k\end{array}\right)([28])$, which goes to 1 asymptotically as $n$ increases if $\lim _{n \rightarrow+\infty} \frac{m}{n}<\frac{1}{2}$. Thus, if $\lim _{n \rightarrow+\infty} \frac{m}{n}<\frac{1}{2}$, then for a random Gaussian matrix $A$, $\left\{\mathbf{x} \mid A \mathbf{x}=A \mathbf{x}_{0}, \mathbf{x} \geq \mathbf{0}\right\}$ would not be a singleton with overwhelming probability no matter how sparse $\mathbf{x}_{0}$ is. This phenomenon is also characterized in [13].

The property that $\left\{\mathbf{x} \mid A \mathbf{x}=A \mathbf{x}_{0}, \mathbf{x} \geq \mathbf{0}\right\}$ is a singleton can also be characterized in both high-dimensional geometry [13] and the null space property of $A$ [20]. We state three equivalent statements in Theorem 2 .

Theorem 2 ([13][20]). The following three properties of $A^{m \times n}$ are equivalent:

- For any nonnegative vector $\mathrm{x}_{0} \in \mathbb{R}^{n}$ with a support size no greater than $k$, the set $\left\{\mathbf{x} \mid A \mathbf{x}=A \mathbf{x}_{0}, \mathbf{x} \geq \mathbf{0}\right\}$ is a singleton.

- The polytope $P$ defined in (II.3) has $n$ vertices and is $k$-neighborly.

- For any $\mathbf{w} \neq \mathbf{0}\left(\mathbf{w} \in \mathbb{R}^{n}\right)$ in the null space of $A$, both the positive support and the negative support of $\mathrm{w}$ have a size of at least $k+1$. 
Note that a polytope $P$ is $k$-neighborly if every set of $k$ vertices spans a face $F$ of $P$. $F$ is a face of $P$ if there exists $\boldsymbol{\alpha}_{F} \in \mathbb{R}^{n}$ and a constant $c$ such that $\boldsymbol{\alpha}_{F}^{T} \mathbf{x}=c, \forall \mathbf{x} \in F$, and $\boldsymbol{\alpha}_{F}^{T} \mathbf{x}<c, \forall \mathbf{x} \notin F$ and $\mathbf{x} \in P$.

[13] (Corollary 4.1) shows that there exists a special partial Fourier matrix $\Omega$ with $2 p+1$ rows such that $\{\mathbf{x} \mid \Omega \mathbf{x}=$ $\left.\Omega \mathbf{x}_{0}, \mathbf{x} \geq \mathbf{0}\right\}$ is a singleton for every nonnegative $p$-sparse signal $\mathbf{x}_{0}$. Here we will show the result is the "best" we can hope for in the sense that a matrix $A$ should have at least $2 p+1$ rows if $\left\{\mathbf{x} \mid A \mathbf{x}=A \mathbf{x}_{0}, \mathbf{x} \geq \mathbf{0}\right\}$ is a singleton for every nonnegative $p$-sparse signal $\mathbf{x}_{0}$.

Proposition 1. For a matrix $A^{m \times n}(m<n)$, if $\{\mathbf{x} \mid A \mathbf{x}=$ $\left.A \mathrm{x}_{0}, \mathrm{x} \geq \mathbf{0}\right\}$ is a singleton for any nonnegative $p$-sparse signal $\mathbf{x}_{0}$, then $m \geq 2 p+1$.

Proof: Pick the first $m+1$ columns of $A$, denoted by $\mathbf{a}_{1}, \mathbf{a}_{2}, \ldots, \mathbf{a}_{m+1} \in \mathbb{R}^{m}$. Since there are $m$ equations and $m+1$ variables $u_{1}, u_{2}, \ldots, u_{m+1}$ in (II.3), then (II.3) admits a nonzero solution.

$$
\sum_{i=1}^{m+1} u_{i} \mathbf{a}_{i}=\mathbf{0}
$$

From Theorem 1 we know that $A \in \mathbf{M}^{+}$, i.e. there exists $\mathbf{h}$ such that $\mathbf{h}^{T} A=\boldsymbol{\beta}^{T}>\mathbf{0}$. Taking the inner product of both sides of (II.3) with $\mathbf{h}$, we have $\sum_{i=1}^{m+1} \beta_{i} u_{i}=0$.

Since $\boldsymbol{\beta}>\mathbf{0}$, from $\sum_{i=1}^{m+1} \beta_{i} u_{i}=0$ we know vector $\mathbf{u}=$ $\left(u_{i}, i=1, \ldots, m+1\right)$ should have both positive and negative terms. Collecting positive and negative terms of $\mathbf{u}$ separatively, we can rewrite (II.3) as follows,

$$
\sum_{i \in I_{+}} u_{i} a_{i}=-\sum_{i \in I_{-}} u_{i} a_{i},
$$

where $I_{+}$is the set of indices of positive terms of $\mathbf{u}$ and $I_{-}$ is the set of indices of negative terms. Note that $\left|I_{+}\right|+\left|I_{-}\right| \leq$ $m+1$. We also have $\sum_{i \in I_{+}} \beta_{i} u_{i}=-\sum_{i \in I_{-}} \beta_{i} u_{i} \triangleq r>\overline{0}$ by multiplying $\mathbf{h}^{T}$ to the left of both sides of (II.4).

Suppose $m \leq 2 p$, then $\left|I_{+}\right|+\left|I_{-}\right| \leq m+1 \leq 2 p+1$, thus we know that $\left|I_{+}\right| \leq p$, or $\left|I_{-}\right| \leq p$, or both hold. Let us first consider the case that $\left|I_{+}\right| \leq p$. Define $B^{n \times n}=\operatorname{diag}(\boldsymbol{\beta})$ and let $D^{m \times n}=A B^{-1}$. Then there is a one-to-one correspondence $\mathbf{z}=B \mathbf{x} \in \mathbb{R}^{n}$ between the two sets $\left\{\mathbf{x} \mid A \mathbf{x}=A \mathbf{x}_{0}, \mathbf{x} \geq \mathbf{0}\right\}$ and $\left\{\mathbf{z} \mid D \mathbf{z}=D \mathbf{z}_{0}, \mathbf{z} \geq \mathbf{0}\right\}$, where $\mathbf{z}_{0}=B \mathbf{x}_{0} \in \mathbb{R}^{n}$. Note that for any nonnegative and $k$ sparse vector $\mathbf{x}, \mathbf{z}=B \mathbf{x}$ is also nonnegative and $k$ sparse. And the converse statement also holds. Since $\left\{\mathbf{x} \mid A \mathbf{x}=A \mathbf{x}_{0}, \mathbf{x} \geq \mathbf{0}\right\}$ is a singleton for every nonnegative $p$-sparse signal $\mathbf{x}_{0}$, then $\left\{\mathbf{z} \mid D \mathbf{z}=D \mathbf{z}_{0}, \mathbf{z} \geq \mathbf{0}\right\}$ is also a singleton for every nonnegative $p$-sparse signal $\mathbf{z}_{0}$. From Theorem $2 \operatorname{Conv}\left(\frac{a_{1}}{\beta_{1}}, \frac{a_{2}}{\beta_{2}}, \ldots, \frac{a_{n}}{\beta_{n}}\right)$ is $p$-neighborly, which implies that for any index set $I$ with $|I|=p$, there exists $\boldsymbol{\eta} \in \mathbb{R}^{m}$ and constant $c$ such that $\boldsymbol{\eta}^{T} a_{i}=\beta_{i} c$ for any $i \in I$, and $\boldsymbol{\eta}^{T} a_{i}<\beta_{i} c$ for all $i \notin I$. We consider specifically an index set $I$, which contains $I_{+}$but does not contain $I_{-}$, and its corresponding vector $\boldsymbol{\eta}$. Taking the inner product of both sides of (II.4) with $\eta$, we would get $r c$ on the left and some value strictly smaller than $r c$ on the right, and reach a contradiction. For the case that $\left|I_{-}\right| \leq p$ we can reach a contradiction through similar arguments, thus $m \geq 2 p+1$ holds.
Sparse recovery problems appear in different fields. Specific problem setup may impose further constraints on the measurement matrix. We are particularly interested in network inference problems, in which the measurement matrix is a 0-1 routing matrix. Network inference problems attempt to extract individual parameters based on aggregate measurements in networks. There has been active research in this area including a wide spectrum of approaches ranging from theoretical reasoning to empirical measurements [11][23][15][30][24].

Since the measurement matrices in network inference problems are 0-1 matrices, the instances when $A$ is a $0-1$ matrix are our main focus. Section II-A and II-B prove that a sparse vector can be the unique nonnegative vector satisfying compressed linear measurements if the measurement matrix is a random Bernoulli matrix or an adjacency matrix of an expander graph. Moreover, the support size of the sparse vector can be proportional to the dimension, in other words, the support size of the unique nonnegative vector is $O(n)$ where $n$ is the dimension, while the provable support size for uniqueness property in [5] is $O(\sqrt{n})$. Besides, for any $\theta \triangleq \lim _{n \rightarrow+\infty} \frac{m}{n}>0$, the support size of a sparse vector that is a unique nonnegative solution can always be $O(n)$, while for Gaussian measurement matrices, with high probability, $\left\{\mathrm{x} \mid A \mathrm{x}=A \mathrm{x}_{0}, \mathrm{x} \geq \mathbf{0}\right\}$ would not be a singleton for any nonnegative $\mathbf{x}_{0}$ (with linearly growing sparsity) if $\theta<\frac{1}{2}$ [13]. This also shows the fundamental difference between 0 1 measurement matrices and well studied Gaussian random measurement matrices.

\section{A. Uniqueness with 0-1 Bernoulli Matrices}

First we consider the uniqueness property with dense 0-1 Bernoulli matrix. The measurement matrix $A$ is an $(m+1) \times n$ measurement matrix, with each element in the first $m$ rows of $A$ being i.i.d. Bernoulli random variables, taking values ' 0 ' with probability $\frac{1}{2}$ and taking values ' 1 ' with probability $\frac{1}{2}$. The last row of $A$ is a $1 \times n$ all ' 1 ' vector. We also assume the fraction ratio $\frac{m}{n}$ is a constant $\theta$ as the dimension $n$ grows. It turns out that as $n$ goes to infinity, with overwhelming probability there exists a constant $\gamma>0$ such that $\left\{\mathbf{x} \mid A \mathbf{x}=A \mathbf{x}_{0}, \mathbf{x} \geq \mathbf{0}\right\}$ is a singleton for any nonnegative $(\gamma n-1)$-sparse signal $\mathbf{x}_{0}$. To see this, we first present the following theorem:

Theorem 3. For any $\theta>0$, there exists a constant $\gamma>0$ such that, with overwhelmingly high probability as $n \rightarrow \infty$, any nonzero vector $\mathrm{w}$ in the null space of the measurement A mentioned above has at least $\gamma n$ negative and at least $\gamma n$ positive elements.

Proof: Let us consider an arbitrary nonzero vector $\mathbf{w} \in$ $\mathbb{R}^{n}$ in the null space of $A$. Let $S$ be the support set for the negative elements of $\mathbf{w}$ and let $S^{c}$ be the support set for the nonnegative elements of $\mathbf{w}$. We now want to argue that, with overwhelmingly high probability, the cardinality $|S|$ of the set $S$ can not be too small.

From the large deviation principle and a simple union bound, for any $\epsilon>0$, with overwhelmingly high probability as $n$ goes to infinity, simultaneously for every column of the 
measurement matrix, the sum of its $(m+1)$ elements will be in the range $\left[\frac{1}{2} \theta(1-\epsilon) n, \frac{1}{2} \theta(1+\epsilon) n\right]$.

Since $A \mathbf{w}=\mathbf{0}$, then $A_{S} \mathbf{w}_{S}+A_{S^{c}} \mathbf{w}_{S^{c}}=\mathbf{0}$, where $A_{S}, \mathbf{w}_{S}$, $A_{S^{c}}$, and $\mathbf{w}_{S^{c}}$ are respectively the part of matrix $A$ and vector w indexed by the sets $S$ and $S^{c}$. Multiplying the $1 \times(m+1)$ row vector $[1,1, \ldots, 1]$ to both sides of this equation, we get

$$
U_{S} \mathbf{w}_{S}+U_{S^{c}} \mathbf{w}_{S^{c}}=0
$$

where $U_{S}$ is an $1 \times|S|$ vector, each component of which represents the sum of the elements from the corresponding column of $A_{S} ; U_{S^{c}}$ is an $1 \times\left|S^{c}\right|$ vector, each component of which represents the sum of the elements from the corresponding column of $A_{S^{c}}$.

From the concentration result of the column sums, we know $U_{S} \mathbf{w}_{S} \geq-\frac{1}{2} \theta(1+\epsilon) n\left\|\mathbf{w}_{S}\right\|_{1}$, and $U_{S^{c}} \mathbf{w}_{S^{c}} \geq \frac{1}{2} \theta(1-$ $\epsilon) n\left\|\mathbf{w}_{S^{c}}\right\|_{1}$. But combining these two inequalities with (II.5), we have $\frac{1}{2} \theta(1-\epsilon) n\left\|\mathbf{w}_{S^{c}}\right\|_{1}-\frac{1}{2} \theta(1+\epsilon) n\left\|\mathbf{w}_{S}\right\|_{1} \leq 0$, which implies

$$
\frac{\left\|\mathbf{w}_{S}\right\|_{1}}{\left\|\mathbf{w}_{S^{c}}\right\|_{1}} \geq \frac{1-\epsilon}{1+\epsilon} .
$$

Now we look at the null space of the measurement matrix $A$. First, notice that the null space of $A$ is a subset of the null space of the matrix $A^{\prime}$ comprising of the first $\theta n$ rows of $A$ subtracted by the last row of $A$ (the all ' 1 ' vector). Then the matrix $A^{\prime}$ is a random \pm 1 Bernoulli measurement matrix, which is known to satisfy the restricted isometry condition. Recall one result about the null space property of a matrix satisfying the restricted isometry condition:

Lemma 2 ([6]). Let $\mathbf{h} \in \mathbb{R}^{n}$ be any vector in the null space of $A^{\prime}$ and let $T_{0}$ be any set of cardinality $q$. Then

$$
\left\|\mathbf{h}_{T_{0}}\right\|_{1} \leq \frac{\sqrt{2} \delta_{2 q}}{1-\delta_{2 q}}\left\|\mathbf{h}_{T_{0}^{c}}\right\|_{1},
$$

where $\delta_{2 q}$ is the restricted isometry constant([8]) for sparse vectors with support set size no bigger than $2 q$, namely, $\delta_{2 q}$ is the smallest positive number such that for any set $T$ with $|T| \leq 2 q$, and any vector $\mathbf{y} \in \mathbb{R}^{n}$, the following holds:

$$
\sqrt{m}\left(1-\delta_{2 q}\right)\|\mathbf{y}\|_{2} \leq\left\|A_{T}^{\prime} \mathbf{y}\right\|_{2} \leq \sqrt{m}\left(1+\delta_{2 q}\right)\|\mathbf{y}\|_{2} .
$$

Reasoning from Lemma 2 and (II.6), after some algebra, we know immediately, for $q=|S|, \delta_{2 q}$ must satisfy

$$
\delta_{2 q} \geq \frac{1-\epsilon}{1-\epsilon+\sqrt{2}(1+\epsilon)} .
$$

We also know there exists a $\gamma>0$ such that for any $q \leq \gamma n$, with overwhelmingly high probability as $n \rightarrow \infty$,

$$
\delta_{2 q}<\frac{1-\epsilon}{1-\epsilon+\sqrt{2}(1+\epsilon)},
$$

thus with overwhelmingly high probability as $n \rightarrow \infty$, the size of the negative support, namely $|S|$, is at least $\gamma n$.

Similarly, we have the same conclusion for the cardinality of the support set of the positive elements for any nonzero vector from the null space of the matrix $A$.

Theorem 3 immediately indicates that $\left\{\mathbf{x} \mid A \mathbf{x}=A \mathbf{x}_{0}, \mathbf{x} \geq\right.$ $\mathbf{0}\}$ is a singleton for all nonnegative $\mathbf{x}_{0}$ that is $\gamma n-1$ sparse. Thus the support size of the unique nonnegative vector can be as large as $O(n)$, while the previous result in [5] is $O(\sqrt{n})$.

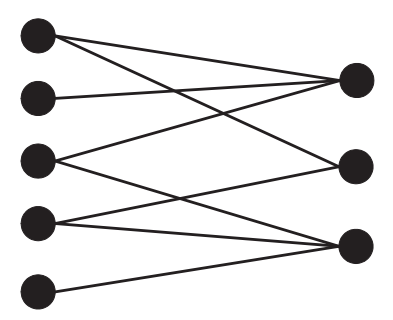

Fig. 1. The bipartite graph corresponding to matrix $A$ in (II.7)

\section{B. Uniqueness with Expander Adjacency Matrices}

Section II-A discusses the singleton property with 0-1 Bernoulli matrices, here we focus on another type of 0 1 matrices where the matrix $A$ is the adjacency matrix of a bipartite expander graph. [4], [20], [29] studied related problems using expander graph with constant left degree. We instead employ a general definition of expander which does not require constant left degree.

Every $m \times n$ binary matrix $A$ is the adjacency matrix of an unbalanced bipartite graph with $n$ left nodes and $m$ right nodes. There is an edge between right node $i$ and left node $j$ if and only if $A_{i j}=1$. Let $d_{j}$ denote the degree of left node $j$, and let $d_{l}$ and $d_{u}$ be the minimum and maximum of left degrees. Define $\rho=d_{l} / d_{u}$, then $0<\rho \leq 1$. For example, the bipartite graph in Fig. 1 corresponds to the matrix $A$ in (II.7). Here $d_{l}=1, d_{u}=2$, and $\rho=0.5$.

$$
A=\left[\begin{array}{lllll}
1 & 1 & 1 & 0 & 0 \\
1 & 0 & 0 & 1 & 0 \\
0 & 0 & 1 & 1 & 1
\end{array}\right]
$$

Definition 2 ([22]). A bipartite graph with $n$ left nodes and $m$ right nodes is an $(\alpha, \delta)$ expander if for any set $S$ of left nodes of size at most $\alpha$, the size of the set of its neighbors $\Gamma(S)$ satisfies $|\Gamma(S)| \geq \delta|E(S)|$, where $E(S)$ is the set of edges connected to nodes in $S$, and $\Gamma(S)$ is the set of right nodes connected to $S$.

Our next main result regarding the singleton property of an adjacency matrix of a general expander is stated as follows.

Theorem 4. For an adjacency matrix $A$ of an $(\alpha, \delta)$ expander with left degrees in the range $\left[d_{l}, d_{u}\right]$, if $\delta \rho>\frac{\sqrt{5}-1}{2} \approx 0.618$, then for any nonnegative $k$-sparse vector $\mathbf{x}_{0}$ with $k \leq \frac{\alpha}{1+\delta \rho} n$, $\left\{\mathrm{x} \mid A \mathrm{x}=A \mathrm{x}_{0}, \mathbf{x} \geq \mathbf{0}\right\}$ is a singleton.

Proof: From Theorem 2, in order to prove that $\{\mathbf{x} \mid A \mathbf{x}=$ $\left.A \mathbf{x}_{0}, \mathbf{x} \geq \mathbf{0}\right\}$ is a singleton for any nonnegative $\frac{\alpha}{1+\delta \rho} n$-sparse vector $\mathbf{x}_{0}$, we only need to argue that for any nonzero $\mathbf{w}$ such that $A \mathbf{w}=\mathbf{0}$, we have $\left|S_{-}\right| \geq \frac{\alpha n}{1+\delta \rho}+1$ and $\left|S_{+}\right| \geq \frac{\alpha n}{1+\delta \rho}+1$, where $S_{-}$and $S_{+}$are negative support and positive support of $\mathbf{w}$ respectively.

We will prove by contradiction. Suppose without loss of generality that there exists a nonzero $\mathbf{w}$ in $\operatorname{Null}(A)$ such that $\left|S_{-}\right|=s \leq \frac{\alpha n}{1+\delta \rho}$, then the set $E\left(S_{-}\right)$of edges connected to nodes in $S_{-}$satisfies $d_{l} s \leq\left|E\left(S_{-}\right)\right| \leq d_{u} s$. Then the set $\Gamma\left(S_{-}\right)$of neighbors of $S_{-}$satisfies

$$
d_{u} s \geq\left|E\left(S_{-}\right)\right| \geq\left|\Gamma\left(S_{-}\right)\right| \geq \delta\left|E\left(S_{-}\right)\right| \geq \delta d_{l} s,
$$

where the third equality comes from the expander property. 
Notice that $\Gamma\left(S_{-}\right)=\Gamma\left(S_{+}\right)=\Gamma\left(S_{-} \cup S_{+}\right)$, otherwise $A w=\mathbf{0}$ does not hold, then $\left|S_{+}\right| \geq \frac{\left|\Gamma\left(S_{+}\right)\right|}{d_{u}}=\frac{\left|\Gamma\left(S_{-}\right)\right|}{d_{u}} \geq$ $\frac{\delta d_{l} s}{d_{u}}=\delta \rho s$.

Now consider the set $S_{-} \cup S_{+}$, we have $\left|S_{-} \cup S_{+}\right| \geq(1+$ $\delta \rho) s$. Pick an arbitrary subset $\tilde{S} \in S_{-} \cup S_{+}$such that $|\tilde{S}|=$ $(1+\delta \rho) s \leq \alpha n$. From expander property, we have

$$
|\Gamma(\tilde{S})| \geq \delta|E(\tilde{S})| \geq \delta d_{l}|\tilde{S}|=\delta \rho(1+\delta \rho) d_{u} s>d_{u} s .
$$

The last inequality holds since $\delta \rho(1+\delta \rho)>1$ provided $\delta \rho>$ $\frac{\sqrt{5}-1}{2}$. But $|\Gamma(\tilde{S})| \leq\left|\Gamma\left(S_{-} \cup S_{+}\right)\right|=\left|\Gamma\left(S_{-}\right)\right| \leq d_{u}$ s. A contradiction arises, which completes the proof.

Corollary 1. For an adjacency matrix $A$ of an $(\alpha, \delta)$ expander with constant left degree $d$, if $\delta>\frac{\sqrt{5}-1}{2}$, then for any nonnegative $k$-sparse vector $\mathbf{x}_{0}$ with $k \leq \frac{\alpha}{1+\delta} n,\{\mathbf{x} \mid A \mathbf{x}=$ $\left.A \mathbf{x}_{0}, \mathbf{x} \geq \mathbf{0}\right\}$ is a singleton.

Theorem 4 together with Corollary 1 is an extension to existing results. Theorem 3.5 of [20] shows that for an $(\alpha, \delta)$ expander with constant left degree $d$, if $d \delta>1$, then there exists a matrix $\tilde{A}$ (a perturbation of $A$ ) such that $\left\{\mathbf{x} \mid \tilde{A} \mathbf{x}=\tilde{A} \mathbf{x}_{0}, \mathbf{x} \geq \mathbf{0}\right\}$ is a singleton for every nonnegative $\delta \alpha n$-sparse $\mathbf{x}_{0}$. Our result instead can directly quantify the sparsity threshold needed for a vector to be a unique solution to compressed measurements induced by $A$, not its perturbation. [4] discussed the success of $\ell_{1}$ recovery of a general vector $\mathbf{x}$ for expanders with constant left degree. If we apply Theorem 1 of [4] to cases where $\mathbf{x}$ is known to be nonnegative, the result can be interpreted as that $\left\{\mathbf{x} \mid A \mathbf{x}=A \mathbf{x}_{0}, \mathbf{x} \geq \mathbf{0}\right\}$ is a singleton for any nonnegative $\frac{\alpha}{2} n$-sparse vector $\mathbf{x}_{0}$ if $\delta>\frac{5}{6} \approx 0.833$. Our result in Corollary 1 implies that if $\delta>\frac{\sqrt{5}-1}{2} \approx 0.618, \mathbf{x}_{0}$ can be $\frac{\alpha}{1+\delta} n$-sparse and still be the unique nonnegative solution.

[27][17] proved that for any $m, n$ and $\delta>0$, there exists an $(\alpha, \delta)$ expander with constant left degree $d$ for some $d$ and $\alpha>0$, and such an expander can be generated through random graphs. There also exist explicit constructions of expander graphs [10]. Combining the results with Corollary 1, for any $m$ and $n$, we can generate an $(\alpha, \delta)$ expander with adjacency matrix $A$ such that $\left\{\mathbf{x} \mid A \mathbf{x}=A \mathbf{x}_{0}, \mathbf{x} \geq \mathbf{0}\right\}$ is a singleton for any nonnegative $k n$-sparse $\mathbf{x}_{0}$, where $k=\frac{\alpha}{1+\delta}>0$. Thus, same as Bernoulli 0-1 matrices, the adjacency matrix $A$ of an $(\alpha, \delta)$ expander has the property that $\left\{\mathbf{x} \mid A \mathbf{x}=A \mathbf{x}_{0}, \mathbf{x} \geq \mathbf{0}\right\}$ is a singleton as long as the support size of $\mathbf{x}_{0}$ is $O(n)$. We further provide an explicit constant $\frac{\alpha}{1+\delta}$ of the ratio of the support size to the dimension. Note that this result is independent of $\frac{m}{n}$, while as discussed earlier, if the matrix has i.i.d. Gaussian entries and $\lim _{n \rightarrow+\infty} \frac{m}{n}<\frac{1}{2},\left\{\mathbf{x} \mid A \mathbf{x}=A \mathbf{x}_{0}, \mathbf{x} \geq \mathbf{0}\right\}$ is not a singleton despite the sparsity of $\mathbf{x}_{0}$.

\section{Unique Positive Semidefinite Solution to AN UNDERDETERMINED SYSTEM}

\section{A. When is Low-rank Positive Semidefinite Solution the Unique Solution?}

Section II studies the case when a sparse nonnegative vector is the only nonnegative solution to the system of compressed linear measurements. Here we extend the problem into the matrix space. Let $X$ be an $n \times n$ matrix decision variable. Let $\mathcal{A}: \mathbb{R}^{n \times n} \rightarrow \mathbb{R}^{m}$ be a linear map, and let $\mathbf{b} \in \mathbb{R}^{m}$. The main optimization problem under study for low-rank matrix recovery is

$$
\begin{array}{ll}
\text { minimize } & \operatorname{rank}(X) \\
\text { subject to } & \mathcal{A}(X)=\mathbf{b} .
\end{array}
$$

In this paper, we are interested in looking at the property of the feasible set $\left\{X^{\prime} \mid \mathcal{A}\left(X^{\prime}\right)=\mathbf{b}\right\}$. Indeed, if there exists a $X^{\prime}$ such that $\mathcal{A}\left(X^{\prime}\right)=\mathbf{b}$, then $X^{\prime}$ plus any matrix in the null space of $\mathcal{A}$ also satisfies $\mathcal{A}\left(X^{\prime}\right)=\mathbf{b}$. However, in applications, one is often interested in recovering a positive semidefinite symmetric matrix $X,\left(X \succeq \mathbf{0}\right.$ and $X \in S^{n}$, where $S^{n}$ is the set of $n \times n$ real symmetric matrices) from compressed observations. To determine a positive semidefinite symmetric matrix $X$, we only need to determine $\frac{n(n+1)}{2}$ unknowns in the upper triangular part of $X$. Thus the linear operator $\mathcal{A}(X)$ in (III.1) is equivalent to an operator $\mathcal{A}^{*}\left(X^{\perp}\right): \mathbb{R}^{\frac{n(n+1)}{2}} \rightarrow \mathbb{R}^{m}$, where $m \leq \frac{n(n+1)}{2}$ and $X^{\perp}$ denotes the upper triangular part of the $n \times n$ symmetric matrix $X$. The null space of $\mathcal{A}^{*}$ is a subset of $\mathbb{R}^{\frac{n(n+1)}{2}}$ such that each point from this set, arranged accordingly as the upper triangular part of an $n \times n$ matrix $Y$, satisfies $\mathcal{A}(Y)=\mathcal{A}^{*}\left(Y^{\perp}\right)=\mathbf{0} \in \mathbb{R}^{m}$.

Now we ask this question, can we uniquely determine the positive semidefinite symmetric matrix $X$ from $\mathcal{A}(X)=\mathbf{b}$, namely can the feasible set $\left\{X^{\prime} \mid \mathcal{A}\left(X^{\prime}\right)=\mathbf{b}, X^{\prime} \succeq\right.$ $\left.\mathbf{0}, X^{\prime} \in S^{n}\right\}$ be a singleton? The next theorem gives an affirmative answer to this question, and shows that if the linear measurement operator satisfies certain conditions and the positive semidefinite symmetric matrix $X$ is of low rank, then the feasible set $\left\{X^{\prime} \mid \mathcal{A}\left(X^{\prime}\right)=\mathbf{b}, X^{\prime} \succeq \mathbf{0}, X^{\prime} \in S^{n}\right\}$ is a singleton, namely $X$ is not only the only low-rank solution, but also the only possible solution.

Theorem 5. Let $X$ be a positive semidefinite symmetric matrix of rank $r$ and $\mathcal{A}^{*}: \mathbb{R}^{\frac{n(n+1)}{2}} \rightarrow \mathbb{R}^{m}$ be a linear operator which operates on the upper triangular part of $X$, where $m<$ $\frac{n(n+1)}{2}$. Then $\left\{X^{\prime} \mid \mathcal{A}\left(X^{\prime}\right)=\mathcal{A}(X), X^{\prime} \succeq \mathbf{0}, X^{\prime} \in S^{n}\right\}$ is a singleton for all $X$ with rank no greater than $r$, if and only if for every non-all-zero symmetric matrix generated from the null space of $\mathcal{A}^{*}$ has at least $r+1$ negative eigenvalues.

Proof: Sufficiency: we first show that if every non-allzero symmetric matrix generated from the null space of $\mathcal{A}^{*}$ has at least $r+1$ negative eigenvalues, then $\left\{X^{\prime} \mid \mathcal{A}\left(X^{\prime}\right)=\right.$ $\left.\mathcal{A}(X), X^{\prime} \succeq \mathbf{0}, X^{\prime} \in S^{n}\right\}$ is a singleton. Suppose instead there exist a $X^{\prime \prime} \in S^{n}$ such that $X^{\prime \prime} \neq X$ and $\mathcal{A}\left(X^{\prime \prime}\right)=$ $\mathcal{A}(X)$, then the upper triangular part of $X^{\prime \prime}-X$ is in the null space of the linear operator $\mathcal{A}^{*}$. By the assumption, we know that $X^{\prime \prime}-X$ has at least $r+1$ negative eigenvalues. Since $X^{\prime \prime}-X$ is a symmetric matrix, its eigenvalues are real. For a matrix, we denote these eigenvalues in an nondecreasing order, namely, $\lambda_{1} \leq \lambda_{2} \leq \cdots \lambda_{n-1} \leq \lambda_{n}$.

By a classical variational characterization of eigenvalues [19], if $A$ and $B$ are both $n \times n$ Hermitian matrices and $B$ has rank at most $r$, then $\lambda_{k}(A+B) \leq \lambda_{k+r}(A)$, for $k=1,2, \ldots, n-r$. By taking $k=1, B=X$ and $A=X^{\prime \prime}-X$, we have $\lambda_{1}\left(X^{\prime \prime}\right)=\lambda_{1}\left(\left(X^{\prime \prime}-X\right)+X\right) \leq \lambda_{r+1}\left(X^{\prime \prime}-X\right)<0$, by the eigenvalue assumption for $X^{\prime \prime}-X$. But then $X^{\prime \prime}$ is not 
a positive semidefinite matrix. This contradiction shows that $X$ is the only element in the the set $\left\{X^{\prime} \mid \mathcal{A}\left(X^{\prime}\right)=\mathcal{A}(X), X^{\prime} \succeq\right.$ 0, $\left.X^{\prime} \in S^{n}\right\}$.

Necessity: we need to show that if there exists a nontrivial symmetric matrix (say $Y$ ), with its upper triangular part from the null space of the linear operator $\mathcal{A}^{*}$, has at most $r$ negative eigenvalues, then we can find an $X$ such that $\left\{X^{\prime} \mid \mathcal{A}\left(X^{\prime}\right)=\right.$ $\left.\mathcal{A}(X), X^{\prime} \succeq \mathbf{0}, X^{\prime} \in S^{n}\right\}$ is not a singleton. Indeed, since $Y$ is a symmetric matrix, it can be diagonalized by some unitary matrix $U$, namely $Y=U \Lambda U^{-1}$, where $\Lambda$ is a diagonal matrix with $\Lambda_{i, i}=\lambda_{i}(Y)$. We then pick $X=U \Lambda^{\prime} U^{-1}$, where $\Lambda^{\prime}$ is a diagonal matrix, and $\Lambda_{i, i}^{\prime}>\max \left\{-\lambda_{i}, 0\right\}$ for $1 \leq i \leq r$ and $\Lambda_{i, i}^{\prime}=0$ for $i>r$. Thus $X$ is a positive semidefinite matrix with rank no larger than $r$ (note that the eigenvalues of $\Lambda^{\prime}$ are not necessarily arranged in nondecreasing order with respect to $i$ ). Then obviously $X+Y=U \Lambda^{\prime \prime} U^{-1}$, where the diagonal entries in the diagonal matrix $\Lambda^{\prime \prime}=\Lambda^{\prime}+\Lambda$ are all nonnegative. Since $Y$ is not a all-zero matrix, $X+Y$ is an element in the set $\left\{X^{\prime} \mid \mathcal{A}\left(X^{\prime}\right)=\mathcal{A}(X), X^{\prime} \succeq \mathbf{0}, X^{\prime} \in S^{n}\right\}$ besides $X$.

Theorem 5 establishes the necessary and sufficient condition for the uniqueness of low-rank positive semidefinite solution under compressed linear measurements. However, checking this condition for a specific set of linear measurements seems to be a hard problem and, in addition, it is not clear whether asymptotically there exist such linear compressed measurements satisfying the given condition. So in Section III-B, we will investigate whether a set of linear measurements (namely the linear measurement $\mathcal{A}^{*}(\cdot)$ ) sampled from a certain distribution will satisfy this condition.

\section{B. The Null Space Analysis of the Gaussian Ensemble}

We say that the linear operator $\mathcal{A}^{*}\left(X^{\perp}\right): \mathbb{R}^{\frac{n(n+1)}{2}} \rightarrow \mathbb{R}^{m}$ is sampled from an independent Gaussian ensemble if its $i$-th $(1 \leq i \leq m)$ operation, denoted by $\mathcal{A}_{i}^{*}\left(X^{\perp}\right): \mathbb{R}^{\frac{n(n+1)}{2}} \rightarrow \mathbb{R}$, is the inner product $\left\langle X, A_{i}\right\rangle=\operatorname{trace}\left(X^{T} A_{i}\right)$, where $A_{i}$ is an $n \times n$ symmetric matrix with independent random elements in its upper triangular part. The diagonal elements of $A_{i}$ are distributed as real Gaussian random variables $N(0,1)$. The offdiagonal elements of $A_{i}$ are distributed as $N\left(0, \frac{1}{2}\right)$. Across the index $i$, the $A_{i}$ 's are also sampled independently. One main result of this paper is stated in the following theorem.

Theorem 6. Consider a linear operator $\mathcal{A}^{*}: \mathbb{R}^{\frac{n(n+1)}{2}} \rightarrow$ $\mathbb{R}^{m}$ sampled from an independent Gaussian ensemble. Let $m=\alpha \times \frac{n(n+1)}{2}$. Then there exists a constant $\alpha<1$, independent of $n$, such that with overwhelming probability as $n$ goes to $\infty$, any nonzero symmetric $n \times n$ square matrix with its upper triangular part from the null space of the linear operator $\mathcal{A}^{*}$ has at least $\xi n$ negative eigenvalues, where $\xi>0$ is a constant that is independent of $n$. Thus with overwhelmingly high probability, any positive semidefinite matrix of rank no larger than $\xi n-1$ will be the singleton in the set $\left\{X^{\prime} \mid \mathcal{A}\left(X^{\prime}\right)=\mathcal{A}(X), X^{\prime} \succeq \mathbf{0}, X^{\prime} \in S^{n}\right\}$.

Note that in Theorem 6 , the constant $\xi$ depends on $\alpha$. Theorem 6 confirms that there indeed exists a sequence of linear operators such that every nonzero element in their null spaces necessarily generates a symmetric matrix having a sufficiently large number $(\xi n)$ of negative eigenvalues. The "guaranteed" number of negative eigenvalues is highly nontrivial in the sense that $\xi n$ grows proportionally with $n$ while the null space for the linear operator $\mathcal{A}^{*}$ has dimension at least $(1-\alpha) \frac{n(n+1)}{2}$, which grows proportionally with $n^{2}$. This seems counterintuitive at first sight: a null space of such a large dimension should have been able to accommodate at least one point which generates a symmetric matrix with very few or even none negative eigenvalues.

The main difficulty in proving Theorem 6 is to show that for all the nonzero symmetric matrices generated from the points in the null space of the random linear operator $\mathcal{A}^{*}$, the claimed fact holds universally with overwhelming probability. This seems to be a daunting job since the null space of every linear operator is a continuous object and there are uncountably many symmetric matrices that can be generated from it. In fact, we have the following probabilistic characterization with a shortened proof for the null space of the linear operator sampled from the independent Gaussian Ensemble.

Lemma 3. If the linear operator $\mathcal{A}^{*}(X): \mathbb{R}^{\frac{n(n+1)}{2}} \rightarrow \mathbb{R}^{m}$ is sampled from independent Gaussian Ensemble, by representing the vectors from the null space of $\mathcal{A}^{*}$ by $\frac{n(n+1)}{2} \times 1$ column vectors, the distribution of its null space is (almost everywhere) equivalent to the distribution of a $\left(\frac{n(n+1)}{2}-m\right)$-dimensional subspace in $\mathbb{R}^{\frac{n(n+1)}{2}}$ whose basis can be represented by $a \frac{n(n+1)}{2} \times\left(\frac{n(n+1)}{2}-m\right)$ matrix $Z$ whose elements are independent Gaussian random variables, $N(0,1)$ for elements in the rows corresponding to the $n$ diagonal elements of $X$ and $N\left(0, \frac{1}{2}\right)$ for elements in the rows corresponding to the $\frac{n(n-1)}{2}$ off-diagonal elements.

Proof: This lemma follows from the fact that a random matrix with zero mean i.i.d. Gaussian distributed entries generates a random subspace whose distribution is rotationally invariant (namely the distribution of that random subspace does not change when it is rotated by a unitary rotation). We also note that if a random subspace has a rotationally invariant distribution, its null space also has a rotationally invariant distribution, which again can be generated by a matrix with zero mean i.i.d. Gaussian distributed entries of appropriate dimensions (with probability 1, the dimension of this null space is $\left.\left(\frac{n(n+1)}{2}-m\right)\right)$. With a normalization for the variance of the Gaussian distributed entries, we have this lemma.

By Lemma 3, the null space of the linear operator $\mathcal{A}^{*}$ sampled from independent Gaussian Ensemble can be represented by $\left\{\mathbf{z} \mid \mathbf{z}=Z \mathbf{w}, \mathbf{w} \in \mathbb{R}^{\frac{n(n+1)}{2}-m}\right\}$, where $Z$ is a $\frac{n(n+1)}{2} \times\left(\frac{n(n+1)}{2}-m\right)$ matrix as mentioned in Lemma 3 .

We should first notice that in order to prove the property that "any nonzero symmetric $n \times n$ square matrix with its upper triangular part from the null space of the linear operator $\mathcal{A}^{*}$ has at least $\xi n$ negative eigenvalue" , we only need to restrict our attention to prove that property for the set of symmetric matrices generated by the set of points $\{\mathbf{z} \mid \mathbf{z}=$ $\left.\frac{1}{\sqrt{n}} Z \mathbf{w}, \mathbf{w} \in \mathbb{R}^{\frac{n(n+1)}{2}-m},\|\mathbf{w}\|_{2}=1\right\}$, in the null space of the linear operator $\mathcal{A}^{*}$. 
Building on this observation, we can proceed to divide the formal proof of Theorem 6 into three steps. Firstly, since we can not show directly our theorem for every point in the null space, instead we first try to discretize the sphere $\left\{\mathbf{w} \mid\|\mathbf{w}\|_{2}=\right.$ $\left.1, \mathbf{w} \in \mathbb{R}^{\frac{n(n+1)}{2}-m}\right\}$ into a finite $\epsilon$-net consisting of a finite number of points on the sphere such that every point in the set $\left\{\mathbf{w} \mid\|\mathbf{w}\|_{2}=1, \mathbf{w} \in \mathbb{R}^{\frac{n(n+1)}{2}-m}\right\}$ is in the $\epsilon$ (in terms of Euclidean distance) neighborhood of at least one point from the $\epsilon$-net. Formally, an $\epsilon$-net is a subset $\mathcal{S} \subset\left\{\mathbf{w} \mid\|\mathbf{w}\|_{2}=\right.$ $\left.1, \mathbf{w} \in \mathbb{R}^{\frac{n(n+1)}{2}-m}\right\}$ such that for every point $\mathbf{t}$ in the set $\left\{\mathbf{w} \mid\|\mathbf{w}\|_{2}=1, \mathbf{w} \in \mathbb{R}^{\frac{n(n+1)}{2}-m}\right\}$, one can find $\mathbf{s}$ in $\mathcal{S}$ such that $\|\mathbf{t}-\mathbf{s}\|_{2} \leq \epsilon$. The following lemma is well known in high dimensional geometry about the size estimate of such a $\epsilon$-net, for example, see [21]:

Lemma 4. There is an $\epsilon$-net $\mathcal{S}$ of the unit sphere of $\mathbb{R} \frac{n(n+1)}{2}-m$ of cardinality less than $\left(1+\frac{2}{\epsilon}\right)^{\frac{n(n+1)}{2}}-m$, which is no larger than $e^{\frac{n(n+1)-2 m}{\epsilon}}$.

Secondly, using the large deviation technique or concentration of measure result, we establish the relevant properties for the symmetric matrices generated from these discrete points on the $\epsilon$-net, e.g., the symmetric matrices have a large number of negative eigenvalues with overwhelming probability. Thirdly, we show how property guarantees on the $\epsilon$-net can be used to establish the null space property for the whole null space of the linear operator $\mathcal{A}^{*}$. Section III-C and III-D are then devoted to completing these steps to prove Theorem 6 .

\section{Concentration for a Single Point}

We take any point $\mathbf{w}$ from the $\epsilon$-net for the set $\left\{\mathbf{w} \mid\|\mathbf{w}\|_{2}=\right.$ $\left.1, \mathbf{w} \in \mathbb{R}^{\frac{n(n+1)}{2}-m}\right\}$ and its corresponding point $\mathbf{z}=\frac{1}{\sqrt{n}} Z \mathbf{w}$ in the null space of the linear operator $\mathcal{A}^{*}$, where $Z$ is the random basis as mentioned in Lemma 3 . We argue that the symmetric matrix $G$ with upper triangular part generated from $\mathbf{z}$ has many negative eigenvalues with overwhelming probability. Obviously with the i.i.d. Gaussian probabilistic model for $Z$, the elements of $G$ are independently Gaussian distributed $N\left(0, \frac{1}{n}\right)$ random variables on the diagonal and independently Gaussian distributed $N\left(0, \frac{1}{2 n}\right)$ on the off-diagonal.

Theorem 7. The smallest $\alpha_{1} n\left(\alpha_{1}<\frac{1}{2}\right)$ eigenvalues of the symmetric matrix $G$ with its upper triangular part generated from $z$ will be upper bounded by $c+\delta$ with overwhelming probability $1-e^{-c_{1} n^{2}}$, where $c$ is a negative number as determined from the semicircular law $\alpha_{1}=\frac{1}{\pi} \int_{-\infty}^{c} \mathbf{1}_{|x|<\sqrt{2}} \sqrt{2-x^{2}} d x, \delta$ is an arbitrarily small positive number, $c_{1}$ is a positive constant as a function of $c$ and $\delta$ but independent of $n$, and 1 is the indicator function.

Proof: Indeed Theorem 7 can be derived from known large deviations or concentration of measure results for the empirical eigenvalue distribution of random symmetric Gaussian matrix [1] [18]. The proof strategy is to show that the probability distributions for eigenvalues where the smallest $\alpha_{1} n$ eigenvalues are not upper bounded by $c+\delta$ will be in a set of small measure. Obviously, $G$ has $n$ real eigenvalues $\left(\lambda_{i}\right)_{1 \leq i \leq n}$ arranged in a nondecreasing order and its spectral measure $\hat{\mu}^{n} \triangleq \frac{1}{n} \sum_{i=1}^{n} \delta_{\lambda_{i}}=\frac{1}{n} \sum_{i=1}^{n} \delta\left(\lambda-\lambda_{i}\right)$, where $\delta(\cdot)$ is the delta function. As in [1], we denote the space of probability measure on $\mathbb{R}$ as $\mathcal{M}_{1}^{+}(\mathbb{R})$ and will endow $\mathcal{M}_{1}^{+}(\mathbb{R})$ with its usual weak topology. [1] then gives the following large deviation result for the empirical eigenvalue distribution for the matrix $G$,

Theorem 8 ([1]). Let $\mu \in \mathcal{M}_{1}^{+}(\mathbb{R})$, define the rate function

$$
I_{1}(\mu)=\frac{1}{2}\left(\int x^{2} d \mu(x)-\Sigma(\mu)\right)-\frac{3}{8}-\frac{1}{4} \log (2),
$$

where $\Sigma(\mu)$ is the non commutative entropy $\Sigma(\mu)=$ $\iint \log (|x-y|) d \mu(x) d \mu(y)$. Then

- $\quad I_{1}$ is well defined over the set $\mathcal{M}_{1}^{+}(\mathbb{R})$ and takes its value in $[0,+\infty)$;

- $I_{1}(\mu)$ is infinite as long as $\mu$ satisfies the following:

* $\int x^{2} d x=+\infty$

* there exists a subset $A$ of $R$ with a positive $\mu$ mass but null logarithmic capacity, i.e. a set $A$ such that $\mu(A)>0$ and

$\gamma(A)=\exp \left\{-\inf _{\nu \in \mathcal{M}_{1}^{+}(\mathbb{R})} \iint \log \left(\frac{1}{|x-y|}\right) d \nu(x) d \nu(y)\right\}=0$

- $I_{1}(\mu)$ is a good rate function, namely $\left\{I_{1}(\mu) \leq M\right\}$ is a compact subset of $\mathcal{M}_{1}^{+}(\mathbb{R})$ for $M \geq 0$.

- $I_{1}$ is a convex function on $\mathcal{M}_{1}^{+}(\mathbb{R})$.

- $I_{1}$ achieves its minimum value at a unique probability measure on $\mathbb{R}$ which is described by the Wigner's Semicircle Law.

- The law of the spectral measure $\hat{\mu}^{n}=\frac{1}{n} \sum_{i=1}^{n} \delta_{\lambda_{i}}$ satisfies a full large deviation principle with good rate function $I_{1}$ and in the scales $n^{2}$, that is, for any open subset $O$ of $\mathcal{M}_{1}^{+}(\mathbb{R})$,

$$
\liminf _{n \rightarrow \infty} \frac{1}{n^{2}} \log \left(P\left(\hat{\mu}^{n} \in O\right)\right) \geq-\inf _{O} I_{1}
$$

for any closed subset $F$ of of $\mathcal{M}_{1}^{+}(\mathbb{R})$,

$$
\limsup _{n \rightarrow \infty} \frac{1}{n^{2}} \log \left(P\left(\hat{\mu}^{n} \in F\right)\right) \leq-\inf _{F} I_{1}
$$

We take $c$ and determine $\alpha_{1}$ from the semicircular law as in the statement of Theorem 7. Then the set $A\left(\hat{\mu}^{n}\right)$ of spectral measures satisfying the statement of Theorem 7 can be denoted by $\left\{\hat{\mu}^{n}: \frac{1}{n} \sum_{i=1}^{n} \mathbf{1}_{\lambda_{i} \leq c+\delta}>\alpha_{1}\right\}$, whose complement (in the set of $n$-dimensional spectral measure) is then $\left\{\hat{\mu}^{n}: \frac{1}{n} \sum_{i=1}^{n} \mathbf{1}_{\lambda_{i} \leq c+\delta} \leq \alpha_{1}\right\}$.

Now we take a continuous function $f$ equal to $\mathbf{1}_{x \leq c}$ over the region $(-\infty, c]$, equal to 0 on $[c+\delta,+\infty)$, and linear in between over the region $[c, c+\delta]$. Then the complement of $A\left(\hat{\mu}^{n}\right)$ is a subset of the following:

$$
\begin{aligned}
\left\{\hat{\mu}^{n}: \frac{1}{n} \sum_{i=1}^{n} f\left(\lambda_{i}\right) \leq \alpha_{1}\right\} & =\left\{\hat{\mu}^{n}: \hat{\mu}^{n}(f) \leq \alpha_{1}\right\} \\
& \subseteq\left\{\mu: \mu(f) \leq \alpha_{1}\right\} \triangleq B(\mu),
\end{aligned}
$$

where $\hat{\mu}^{n}=\frac{1}{n} \sum_{i=1}^{n} \delta_{\lambda_{i}}, \mu \in \mathcal{M}_{1}^{+}$, and $\mu(f)$ is the integral of the function $f$ over $\mu$. 
This set $B(\mu)$ is closed for the weak topology and so we can apply the large deviation principle as in [1] to get that

$$
\limsup _{n \rightarrow \infty} \frac{1}{n^{2}} \log P\left(\left\{\frac{1}{n} \sum_{i=1}^{n} \mathbf{1}_{\lambda_{i} \leq c+\delta} \leq \alpha_{1}\right\}\right) \leq-\inf _{B(\mu)} I,
$$

with $I$ as defined in Theorem 8 . However, from the definition of $\alpha_{1}$ and the function $f$, we simply know that the semi-circle law does not belong to the set $B(\mu)$ and so we can conclude that $\inf _{B(\mu)} I>0$. This is because the rate function $I$ is a good rate function which achieves its unique minimum at the semicircle law. So in summary, we can take $c_{1}$ in the statement of this theorem as $\inf _{B(\mu)} I$.

Following Theorem 7, we know that with overwhelming probability, the symmetric matrix generated from a single point on the $\epsilon$-net will be very likely to have a large number (proportional to $n$ ) of negative eigenvalues. In Section III-D, we will show how to synthesize the results for isolated points so that we can prove the eigenvalue claim for the null space of the linear operator $\mathcal{A}^{*}$.

\section{Concentration for the Null Space: $\epsilon$-net Analysis}

Building on the concentration results for the single point on the $\epsilon$-net, we now begin proving the claims in Theorem 6 for all the possible symmetric matrices generated from the set $\left\{\mathbf{z} \mid \mathbf{z}=Z \mathbf{w}, \mathbf{w} \in \mathbb{R}^{\frac{n(n+1)}{2}-m}\right\}$, where $Z$ is a $\frac{n(n+1)}{2} \times$ $\left(\frac{n(n+1)}{2}-m\right)$ matrix as mentioned in Lemma 3 .

First, we make a simple observation regarding every point $\mathbf{w}$ on the Euclidean sphere $\left\{\mathbf{w} \mid\|\mathbf{w}\|_{2}=1, \mathbf{w} \in \mathbb{R}^{\frac{n(n+1)}{2}-m}\right\}$.

Since $\mathcal{S}$ is an $\epsilon$-net on the sphere, we can find a point $\mathbf{w}_{0} \in$ $\mathcal{S}$ with $\left\|\mathbf{w}_{0}\right\|_{2}=1$ such that $\left\|\mathbf{w}-\mathbf{w}_{0}\right\|_{2} \leq \epsilon$. For the error term $\mathbf{w}-\mathbf{w}_{0}$, we can still find a point $\mathbf{w}_{1}$ on the $\epsilon$-net $\mathcal{S}$ such that $\left\|\mathbf{w}-\mathbf{w}_{0}-\right\| \mathbf{w}-\mathbf{w}_{0}\left\|_{2} \mathbf{w}_{1}\right\|_{2} \leq \epsilon\left\|\mathbf{w}-\mathbf{w}_{0}\right\|_{2} \leq \epsilon^{2}$. By iterating this process, we get that any $\mathbf{w}$ on the unit Euclidean sphere can be expressed as

$$
\mathbf{w}=\mathbf{w}_{0}+\sum_{i=1}^{\infty} t_{i} \mathbf{w}_{i}
$$

where $\left|t_{i}\right| \leq \epsilon^{i}$ for $i \geq 1$ and $\mathbf{w}_{i} \in \mathcal{S}$ for $i \geq 0$.

Before we proceed further to look at the spectrum of the symmetric matrix $B_{\mathbf{w}}$ generated from $Z \mathbf{w}$, we state the following theorem by Hoffmann and Wielandt [19].

Theorem 9 ([19]). Let $A, E \in M_{n}$, assume that $A$ and $A+E$ are both normal, let $\lambda_{1}, \ldots, \lambda_{n}$ be the eigenvalues of $A$ in some given order, and let $\hat{\lambda}_{1}, \ldots, \hat{\lambda}_{n}$ be the eigenvalues of $A+E$ in some order. Then there exists a permutation $\sigma(i)$ of the integers $1,2, \ldots, n$ such that $\left(\sum_{i=1}^{n}\left|\hat{\lambda}_{\sigma_{i}}-\lambda_{i}\right|^{2}\right)^{\frac{1}{2}} \leq\|E\|_{2}$.

Now we can give a closer study of the $n \times n$ symmetric matrix $B_{\mathbf{w}}$ generated from $\frac{1}{\sqrt{n}} Z \mathbf{w}$. From the $\epsilon$-net decomposition (III.2), it follows that $B_{\mathbf{w}}=B_{\mathbf{w}_{0}}+\sum_{i=1}^{n} t_{i} B_{\mathbf{w}_{i}}$, where $B_{\mathbf{w}_{i}}$ is the symmetric matrix generated from $\frac{1}{\sqrt{n}} Z \mathbf{w}_{i}$ for $i \geq 0$.

Since we can thus view $B_{\mathbf{w}}$ as $B_{\mathbf{w}_{0}}$ plus some perturbation, using Theorem 9, there exists a permutation $\sigma$ of the integers
$1,2, \ldots, n$ such that

$$
\left(\sum_{i=1}^{n}\left|\hat{\lambda}_{\sigma_{i}}-\lambda_{i}\right|^{2}\right)^{\frac{1}{2}} \leq\left\|\sum_{i=1}^{n} t_{i} B_{\mathbf{w}_{i}}\right\|_{2},
$$

where $\hat{\lambda}_{i}, 1 \leq i \leq n$, and $\lambda_{i}, 1 \leq i \leq n$, are the eigenvalues of the $B_{\mathbf{w}}$ and $B_{\mathbf{w}_{0}}$ arranged in an increasing order, respectively.

But from the triangular inequality, we know

$$
\begin{aligned}
\left\|\sum_{i=1}^{n} t_{i} B_{\mathbf{w}_{i}}\right\|_{2} & \leq \sum_{i=1}^{n}\left|t_{i}\right|\left\|B_{\mathbf{w}_{i}}\right\|_{2} \\
& \leq \sum_{i=1}^{n} \epsilon^{i} C_{1} \sqrt{n} \leq \frac{\epsilon C_{1} \sqrt{n}}{1-\epsilon}
\end{aligned}
$$

where we use the condition that, with overwhelmingly high probability as $n \rightarrow \infty,\left\|B_{\mathbf{w}^{\prime}}\right\|_{2}$ is upper bounded by $C_{1} \sqrt{n}$ simultaneously for all $\mathbf{w}^{\prime} \in \mathcal{S}$ by choosing $\alpha=\frac{2 m}{n(n+1)}$ appropriately (which we will show soon), where $C_{1}^{n(n+1)}$ $\sqrt{\left(\mathbb{E}\left\|B_{\mathbf{w}^{\prime}}\right\|_{2}^{2}\right) / n}$ is a chosen constant. Note that $C_{1}$ can be a constant because $\mathbb{E}\left\|B_{\mathbf{w}^{\prime}}\right\|_{2}^{2}$ scales as $O(n)$ and is the same for every $\mathbf{w}^{\prime} \in \mathcal{S}$.

We pick $c<0$, small enough $\delta>0$ such that $c+\delta<0$, and let $\alpha_{1}$ be determined from $c>0$ as in Theorem 7. Assume also that the smallest $\alpha_{1} n$ eigenvalues of $B_{\mathbf{w}_{0}}$ is upper bounded by $c+\delta$. We can then officially argue that the number, say $k$, of negative eigenvalues of $B_{\mathrm{w}}$, can not be small. In particular, we will upper bound $\left(\alpha_{1} n-k\right)$ for $B_{\mathbf{w}}$. For whatever ordering $\sigma$ the eigenvalues of $B_{\mathbf{w}}$ take,

$$
\sum_{i=1}^{n}\left|\hat{\lambda}_{\sigma_{i}}-\lambda_{i}\right|^{2} \geq\left(\alpha_{1} n-k\right)|c+\delta|^{2},
$$

because at least $\left(\alpha_{1} n-k\right)$ negative eigenvalues (which are all smaller than $c+\delta$ ) of $B_{\mathbf{w}_{0}}$ will be matched to nonnegative eigenvalues of $B_{\mathbf{w}}$ in Theorem 9 .

Connecting (III.3), (III.4) and (III.5), if $\left(\alpha_{1} n-k\right) \geq 0$, (otherwise $k$ already nicely bounded), then $\sqrt{\left(\alpha_{1} n-k\right)|c+\delta|^{2}} \leq$ $\epsilon C_{1} \sqrt{n} /(1-\epsilon)$, namely, $k \geq \alpha_{1} n-\frac{\epsilon^{2} C_{1}^{2} n}{(1-\epsilon)^{2}|c+\delta|^{2}}$. If we pick $\epsilon>0$ small enough, the number of negative eigenvalues of $B_{\mathbf{w}}$ will be proportionally growing with $n$.

We have shown that, if for all $\mathbf{w}^{\prime} \in \mathcal{S},\left\|B_{\mathbf{w}^{\prime}}\right\|_{2}$ is upper bounded by $C_{1} \sqrt{n}$, and the smallest $\alpha_{1} n$ eigenvalues of $B_{\mathbf{w}^{\prime}}$ are upper bounded by $c+\delta$, then the number of negative eigenvalues of every nonzero matrix from the null space would be at least $k \geq \alpha_{1} n-\frac{\epsilon^{2} C_{1}^{2} n}{(1-\epsilon)^{2}|c+\delta|^{2}}$. However, these two regularity conditions indeed will happen with overwhelming probability by choosing $\alpha=\frac{2 m}{n(n+1)}$ appropriately.

From Theorem 7 , for $a \mathbf{w}^{\prime} \in \mathcal{S}$, with probability at most $e^{-c_{1} n^{2}}$, (where $c_{1}>0$ is a constant determined solely by $c<0$ and $\delta>0$ ), the smallest $\alpha_{1} n$ eigenvalues of $B_{\mathbf{w}^{\prime}}$ are upper bounded by $c+\delta$. Also, by a standard Chernoff bound or a large deviation argument, for $a \mathbf{w}^{\prime} \in \mathcal{S},\left\|B_{\mathbf{w}^{\prime}}\right\|_{2}>C_{1} \sqrt{n}$ happens with probability at most $e^{-c_{2} n^{2}}$, where $c_{2}>0$ is a constant that depends only on $C_{1}$.

Now by a union bound over the $\epsilon$-net, which has at most $e^{\frac{n(n+1)-2 m}{\epsilon}}$ discrete points, the two regularity conditions will happen with overwhelming probability, if $\frac{n(n+1)-2 m}{\epsilon}-c_{1} n^{2}<$ 0 and $\frac{n(n+1)-2 m}{\epsilon}-c_{2} n^{2}<0$, namely, $\alpha=\frac{{ }^{\epsilon} m}{n(n+1)}>1-\epsilon c_{2}$ 
and $\alpha=\frac{2 m}{n(n+1)}>1-\epsilon c_{1}$. We can achieve this by taking $m$ large enough, but still keeping $\alpha=\frac{2 m}{n(n+1)}<1$, since we have $\epsilon>0, c_{1}>0$ and $c_{2}>0$.

It is necessary to clarify the relationships between the variables $c, \delta, \alpha_{1}, c_{1}, C_{1}, c_{2}, \epsilon$ and $\alpha$. We first pick $c<0$ and then pick $\delta>0$ as another small number such that $c+\delta<0$. As we state in Theorem 7 and its proof, $\alpha_{1}>0$ is determined completely by $c$ from the semicircular law; $c_{1}$ can be solely characterized as a function of $c$ and $\delta . C_{1}>\sqrt{\frac{\mathbb{E}\left\|B_{\mathbf{w}^{\prime}}\right\|_{2}^{2}}{n}}$ is another independent constant which we pick, and the exponent $c_{2}$ is determined solely from $C_{1}$ using the large deviation principle. We then pick $\epsilon>0$ such that $\alpha_{1}>\frac{\epsilon^{2} C_{1}^{2}}{(1-\epsilon)^{2}|c+\delta|^{2}}$. In justifying the two regularity conditions, we finally determine $\alpha=\frac{2 m}{n(n+1)}$ appropriately to let the union bound exponent overridden by the two decaying exponents $c_{1}$ and $c_{2}$.

Thus, we have arrived at a complete proof of Theorem 6 .

\section{Simulation}

In the vector case, we generate a random 0-1 matrix $A^{m \times n}$ with i.i.d. entries and empirically study the uniqueness property and the success of $\ell_{1}$ minimization for nonnegative vectors with different sparsity. Each entry of $A$ takes value 1 with probability 0.2 and value 0 with probability 0.8 . The size of $A$ is $50 \times 200$ and $100 \times 200$ respectively. For a sparsity $k$, we select a support set $S$ with size $|S|=k$ uniformly at random, and generate a nonnegative vector $\mathrm{x}_{0}$ on $S$ with i.i.d. entries uniformly on the unit interval. Then we check whether $U \triangleq\left\{\mathbf{x} \mid A \mathbf{x}=A \mathbf{x}_{0}, \mathbf{x} \geq \mathbf{0}\right\}$ is singleton. This can be realized as follows. We minimize and maximize the same objective function $\mathbf{d}^{T} \mathbf{x}$ over $U$, where $\mathbf{d}$ is a random vector in $\mathbb{R}^{n}$. Note that if $U$ is not a singleton, then the set $\left\{\mathbf{d} \in \mathbb{R}^{n} \mid \mathbf{d}^{T} \mathbf{x}=\mathbf{d}^{T} \mathbf{x}_{0}, \forall \mathbf{x} \in U\right\}$ has measure 0. Thus the probability that the minimizer and the maximizer are the same when $U$ is not a singleton is 0 . We generate several different d's and claim $U$ to be singleton if the minimizer and the maximizer are the same for every $\mathbf{d}$. For each instance, we also check whether $\ell_{1}$ minimization can recover $\mathrm{x}_{0}$ from $A \mathbf{x}_{0}$ or not. Under a given sparsity $k$, we generate $200 \mathrm{x}_{0}$ 's and repeat the above procedure 200 times.

We fix $n=200$, and $m$ is 50 in Fig. 2(a) and 100 in Fig. 2(b). When $\frac{m}{n}$ increases from $\frac{1}{4}$ to $\frac{1}{2}$, the support size of a sparse vector which is a unique nonnegative solution increases from $0.05 n$ to $0.19 n$. Note that when $\frac{m}{n}=\frac{1}{2}$, for this $0-1$ matrix, the singleton property still exists linearly in $n$, while for a random Gaussian matrix, with overwhelming probability no vector can be a unique nonnegative solution. Besides, the thresholds where the singleton property breaks down and where the fully recovery of $\ell_{1}$ minimization breaks down are quite close.

In the matrix case, we generate a $40 \times 40$ matrix $G$ such that all the elements are i.i.d. $N(0,1)$, then $A=\frac{1}{2}\left(G+G^{T}\right)$ has its diagonal elements distributed as $N(0,1)$ and offdiagonal elements distributed as $N\left(0, \frac{1}{2}\right)$. We generate $m$ such matrices $A_{i}$ 's as the linear operator $\mathcal{A}^{*}, m$ is 500 and 600 respectively for comparison. $X$ is a low-rank positive semidefinite symmetric matrix. We increase the rank of $X$ from 0 to $0.4 n$, and for each fixed rank, generate $200 X$ 's randomly. For each $X$, we minimize and maximize the same objective function $\left\langle D, X^{\prime}\right\rangle$ over the set $V \triangleq\left\{X^{\prime} \mid \mathcal{A}\left(X^{\prime}\right)=\right.$ $\left.\mathcal{A}(X), X^{\prime} \succeq \mathbf{0}, X^{\prime} \in S^{n}\right\}$, where $D$ is random matrix with i.i.d. $N(0,1)$ entries. Similarly to the vector case, if $V$ is not a singleton, then the set $\left\{D \mid\left\langle D, X^{\prime}\right\rangle=\langle D, X\rangle, \forall X^{\prime} \in V\right\}$ has measure 0 . Thus the probability that the minimizer and the maximizer are the same when $V$ is not a singleton is 0 . We generate several different $D$ 's and claim the set $V$ to be a singleton if the minimizer and the maximizer of $\left\langle D, X^{\prime}\right\rangle$ from the set $\left\{X^{\prime} \mid \mathcal{A}\left(X^{\prime}\right)=\mathcal{A}(X), X^{\prime} \succeq \mathbf{0}, X^{\prime} \in S^{n}\right\}$ are the same for every $D$. As indicated by Fig. 3, when $m=500$, the singleton property holds if $\operatorname{rank}(X)$ is at most 2 , which is $0.05 n$. When $m$ increases to 600 , the singleton property holds if $\operatorname{rank}(X)$ is at most 8 , which is $0.2 n$.

\section{CONClusion}

This paper studies the phenomenon that an underdetermined system admits a unique nonnegative vector solution or a unique positive semidefinite matrix solution. This uniqueness property can potentially lead to more efficient sparse recovery algorithms. We show that only for a class of matrices with a row span intersecting the positive orthant that $\{\mathbf{x} \mid A \mathbf{x}=$ $\left.A \mathbf{x}_{0}, \mathbf{x} \geq \mathbf{0}\right\}$ could possibly be a singleton if $\mathbf{x}_{0}$ is sparse enough. Among these matrices, we are interested in $0-1$ matrices which fit the setup of network inference problems. For Bernoulli 0-1 matrices, we prove that with high probability the unique solution property holds for all $k$-sparse nonnegative vectors where $k$ is $O(n)$, instead of the previous result $O(\sqrt{n})$. For the adjacency matrix of a general expander, the same phenomenon exists and we further provide a closed-form constant ratio of $k$ to $n$. One future direction is to obtain uniqueness property threshold for a given measurement matrix. So far, we have only discussed the ideally sparse nonnegative vectors, but we would also like to consider recovering approximately sparse nonnegative signal vectors. In approximate sparse recovery problems, instead of being a singleton, the feasible set can contain an infinite number of solutions, but we conjecture its measure is "small".

For the matrix case, we develop a necessary and sufficient condition for a linear compressed operator to admit a unique feasible positive semidefinite matrix solution. We further show that this condition will be satisfied with overwhelmingly high probability for a randomly generated Gaussian linear compressed operator with vastly different approaches from those used in vector case. Computing explicitly the threshold $\xi$ as a function of $\alpha$, for the uniqueness property to happen will be one part of future works.

\section{REFERENCES}

[1] G. B. Arous and A. Guionnet, "Large deviations for Wigner's law and Voiculescu's non-commutative entropy," Probability Theory and Related Fields, vol. 108, no. 4, pp. 517-542, 1997.

[2] R. Baraniuk, "Compressive sensing," IEEE Signal Process. Mag., vol. 24, no. 4, pp. 118-121, July 2007.

[3] R. Berinde, A. Gilbert, P. Indyk, H. Karloff, and M. Strauss., "Combining geometry and combinatorics: a unified approach to sparse signal recovery," Preprint, 2008.

[4] R. Berinde and P. Indyk, "Sparse recovery using sparse random matrices," MIT-CSAIL Technical Report, 2008. 


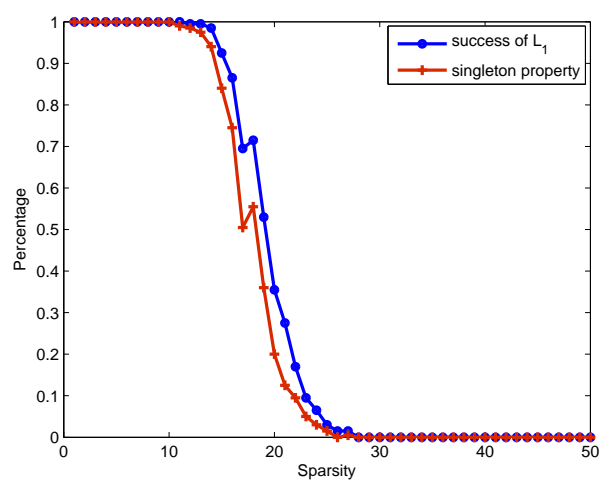

(a) $50 \times 2000-1$ matrix

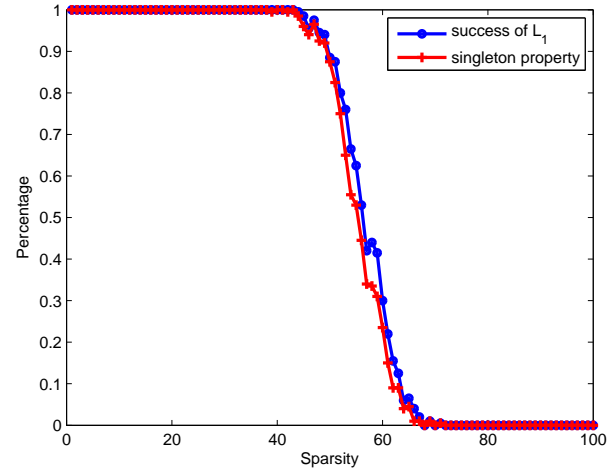

(b) $100 \times 2000-1$ matrix

Fig. 2. Comparison of $\ell_{1}$ recovery and singleton property for (a) $50 \times 2000-1$ matrix and (b) $100 \times 2000-1$ matrix

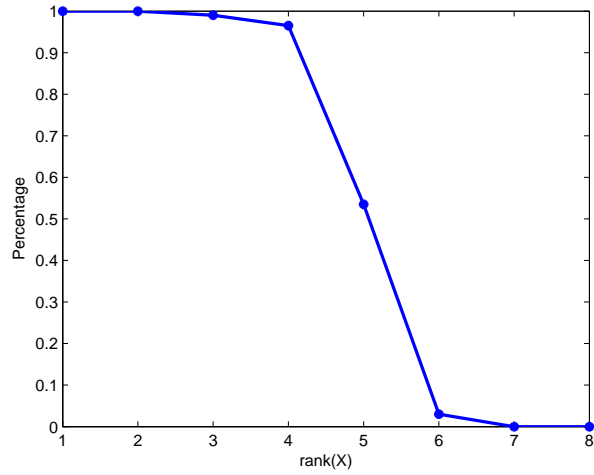

(a) $m=500$

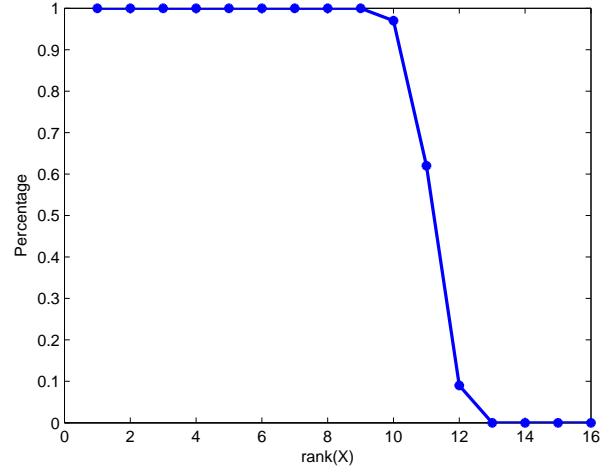

(b) $m=600$

Fig. 3. System of $m$ measurements admitting a unique $40 \times 40$ semidefinite matrix solution (a) $m=500$ (b) $m=600$

[5] A. Bruckstein, M. Elad, and M. Zibulevsky, "On the uniqueness of nonnegative sparse solutions to underdetermined systems of equations," IEEE Trans. Inf. Theory, vol. 54, no. 11, pp. 4813-4820, Nov. 2008.

[6] E. J. Candès, "The restricted isometry property and its implications for compressed sensing," Compte Rendus de l'Academie des Sciences, pp. 589-592, 2008

[7] E. Candès and T. Tao, "Decoding by linear programming," IEEE Trans. Inf. Theory, vol. 51, no. 12, pp. 4203-4215, Dec. 2005.

[8] _ "Near-optimal signal recovery from random projections: Universal encoding strategies?" IEEE Trans. Inf. Theory, vol. 52, no. 12, pp. 54065425, Dec. 2006.

[9] E. Candès, "Compressive sampling," in Proc. International Congress of Mathematics, 2006, pp. 1433-1452.

[10] M. Capalbo, O. Reingold, S. Vadhan, and A. Wigderson, "Randomness Conductors and Constant degree Expansions beyond the degree 2 Barrier," in Proc. ACM STOC, 2002, pp. 659-668.

[11] A. Coates, A. Hero III, R. Nowak, and B. Yu, "Internet tomography," IEEE Signal Process. Mag., vol. 19, no. 3, pp. 47-65, May 2002.

[12] D. L. Donoho and J. Tanner, "Sparse nonnegative solution of underdetermined linear equations by linear programming," in Proc. Natl. Acad. Sci. U.S.A., vol. 102, no. 27, 2005, pp. 9446-9451.

[13] — "Counting the faces of randomly-projected hypercubes and orthants, with applications," Discrete Comput. Geom., 2008.

[14] D. Donoho, "Compressed sensing," IEEE Trans. Inf. Theory, vol. 52, no. 4, pp. 1289-1306, April 2006.

[15] N. Duffied, "Network tomography of binary network performance characteristics," IEEE Trans. Inf. Theory, vol. 52, no. 12, pp. 5373-5388, Dec. 2006

[16] M. Fazel, "Matrix rank minimization with applications," Ph.D. dissertation, Stanford University, 2002.

[17] J. Feldman, T. Malkin, R. A. Servedio, C. Stein, and M. J. Wainwright, "LP decoding corrects a constant fraction of errors," IEEE Trans. Inf. Theory, vol. 53, no. 1, pp. 82-89, 2007.
[18] A. Guionnet and O.Zeitouni, "Concentration of the spherical measure for large matrices," Elect. Commu. in Probab., pp. 119-136, 2000.

[19] R. A. Horn and C. R. Johnson, Matrix Analysis. New York: Cambridge University Press, 1985.

[20] M. A. Khajehnejad, A. G. Dimakis, W. Xu, and B. Hassibi, "Sparse recovery of positive signals with minimal expansion," Preprint, 2009.

[21] M. Ledoux, The Concentration of Measure Phenomenon. Providence: American Mathematical Society, 2000.

[22] M. G. Luby, M. Mitzenmacher, M. A. Shokrollahi, and D. A. Spielman, "Analysis of low density codes and improved designs using irregular graphs," in Proc. ACM STOC, 1998, pp. 249-258.

[23] H. Nguyen and P. Thiran, "Using end-to-end data to infer lossy links in sensor networks," in Proc. IEEE Infocom, Apr. 2006, pp. 1-12.

[24] H. X. Nguyen and P. Thiran, "Network loss inference with second order statistics of end-to-end flows," in Proc. ACM SIGCOMM, 2007, pp. 227240.

[25] B. Recht, M. Fazel, and P. Parrilo, "Guaranteed minimum rank solutions of matrix equations via nuclear norm minimization," Submitted. Preprint Available at http://www.ist.caltech.edu/ brecht/publications.html.

[26] B. Recht, W. Xu, and B. Hassibi, "Necessary and sufficient conditions for success of the nuclear norm heuristic for rank minimization," in Proc. IEEE CDC, 2008.

[27] M. Sipser and D. Spielman, "Expander codes," IEEE Trans. Inf. Theory, vol. 42, no. 6, pp. 1710-1722, 1996.

[28] J. Wendel, "A problem in geometric probability," Mathematica Scandinavica, no. 11, pp. 109-111, 1962.

[29] W. Xu and B. Hassibi, "Efficient compressive sensing with deterministic guarantees using expander graphs," in Information Theory Workshop, 2007. ITW '07. IEEE, Sept. 2007, pp. 414-419.

[30] Y. Zhang, M. Roughan, C. Lund, and D. Donoho, "An informationtheoretic approach to traffic matrix estimation," in Proc. ACM SIGCOMM, Aug. 2003, pp. 301-312.

[31] Y. Zhao, Y. Chen, and D. Bindel, "Towards unbiased end-to-end network diagnosis," in Proc. ACM SIGCOMM, 2006, pp. 219-230. 